\title{
Cateterismo Cardíaco, Diagnóstico (Angiografia) e Terapêutico (Angioplastia) na Doença Arterial Coronária dos Pacientes Diabéticos
}

\begin{abstract}
RESUMO
O rastreamento, o diagnóstico e a revascularização da doença arterial coronária (DAC) no diabetes tipo 2 representam enormes desafios para a prática médica. Neste contexto, o cateterismo cardíaco diagnóstico (angiografia) e terapêutico (angioplastia) são recursos fundamentais na avaliação clínica e manejo da aterosclerose coronária. Avaliações das particularidades anatômicas da DAC no diabetes são detalhadamente caracterizadas pela angiografia coronária, associada ou não ao ultra-som intracoronário. Por outro lado, a resposta pior aos procedimentos de revascularização (angioplastia e cirurgia) da DAC nos diabéticos representa umas das áreas de maior investigação clínica contemporânea. A despeito das controvérsias, cerca de um quarto dos pacientes submetidos a angioplastia e um terço dos pacientes submetidos a cirurgia são diabéticos. Dois grandes estudos multicêntricos randomizados, em andamento, investigam o melhor manejo da DAC nos diabéticos. O BARI 2D está randomizando pacientes com DAC assintomáticos ou com sintomas leves para tratamento clínico ou revascularização (angioplastia ou cirurgia, conforme melhor julgamento clínico). O FREEDOM está randomizando pacientes diabéticos estáveis com DAC multiarterial, para angioplastia com stent farmacológico ou cirurgia, com ou sem uso de circulação extra-corpórea. Enquanto as evidências não estão disponíveis, a prática médica atual é balanceada por um conjunto de variáveis na decisão sobre a melhor alternativa de revascularização. Condições que favorecem a angioplastia: estenoses curtas, vasos grandes, ausência de estenose na artéria descendente anterior (ADA), cirurgia de revascularização prévia e risco cirúrgico elevado. Condições que favorecem a indicação de cirurgia: estenoses longas, vasos pequenos, presença de estenose da ADA e cirurgia valvar associada. (Arq Bras Endocrinol Metab 2007;51/2:299-304)
\end{abstract}

Descritores: Doença arterial coronária; Diabetes mellitus; Cateterismo cardíaco; Angiografia; Angioplastia.

\begin{abstract}
Coronary Angiography and Angioplasty in Diabetic Patients.

Screening, diagnosis and revascularization of coronary artery disease (CAD) in type 2 diabetes mellitus are major challenges for current clinical practice. Diagnostic (angiography) and therapeutic (angioplasty) cardiac catheterization are important resources for the clinical assessment and management of coronary atherosclerosis. Anatomic peculiarities of CAD in diabetics can be well characterized by angiography, associated or not by intravascular ultrasound. The worse outcome following coronary revascularization procedures, either angioplasty or surgery, in diabetic is one of the main fields of clinical research. In spite of controversies, about one quarter of angioplasty and one third of surgical revascularization procedures are performed in diabetics. Two ongoing, large, randomized, multicentric trials are investigating the best management of CAD in diabetics. The BARI 2D trial is randomizing asymptomatic or mildly symptomatic patients with CAD for either medical therapy or revascularization (angioplasty or surgery, according to the best clinical judgment). The FREEDOM trial is randomizing stable patients with multivessel CAD for either angioplasty with drug eluting stents or surgery, with or without extracorporeal circulation. While the evidences are not available, in order to decide on the best revascularization procedure for individual patients, medical practice has been balanced according to a number of variables. Conditions that favor angioplasty: short lesions, lesions in large vessels, absence of left anterior descending artery disease, previous coronary bypass surgery and high surgical risk due to co-morbidities. Conditions that favor surgery: long lesions, lesions in small vessels, presence of left anterior descending artery disease and need for associated valve surgery. (Arq Bras Endocrinol Metab 2007;51/2:299-304)
\end{abstract}

Keywords: Coronary artery disease; Diabetes mellitus; Cardiac catheterization; Angiography; Angioplasty

\section{revisão}

\author{
VALTER C. LIMA
}

Disciplina de Cardiologia, UNIFESP-EPM, e Serviço de Hemodinâmica e Cardiologia Intervencionista do Hospital São Paulo - SPDM e Hospital do Rim e Hipertensão - FOR, São Paulo, SP. 
$\triangle$ DOENÇA ARTERIAL CORONÁRIA (DAC) é duas a quaLtro vezes mais comum nos diabéticos. Além disso, numerosos aspectos próprios da aterosclerose nesses pacientes fazem com que a DAC tenha comportamento clínico, resposta ao tratamento e prognóstico piores do que em pacientes não-diabéticos. A abordagem diagnóstica ainda é dificultada pela coexistência de disfunção microvascular, hipertrofia ventricular esquerda e neuropatia, condições que comprometem a acurácia dos testes diagnósticos não-invasivos, tais como o teste ergométrico, a cintilografia miocárdica e a ecocardiografia com estresse farmacológico.

Entretanto, o rastreamento de DAC em pacientes diabéticos é uma iniciativa comum dos clínicos, pois a mesma tem prevalência epidêmica, sendo a principal causa de morte nesses pacientes. O diagnóstico precoce de DAC em pacientes diabéticos assintomáticos resulta em marcada redução de eventos cardiovasculares. Esta redução de eventos é obtida tanto pela instituição de medidas terapêuticas farmacológicas (drogas anti-trombóticas, anti-isquêmicas e antiateroscleróticas) e não-farmacológicas (modificação do estilo de vida), como pelos procedimentos de revascularização miocárdica (angioplastia e cirurgia).

Entretanto, não há diretrizes baseadas em evidências que recomendem o rastreamento de DAC em diabéticos assintomáticos. As recomendações da American Diabetes Association (ADA), publicadas em 1998 (1), são baseadas apenas em consenso de especialistas e não foram modificadas pelas recomendações globais de diagnóstico e tratamento do diabetes, revisadas em outubro de 2005 e publicadas em janeiro de 2006 (2). A ADA recomenda que apenas os diabéticos tipo 2 assintomáticos com dois ou mais fatores de risco para aterosclerose (dislipidemia, tabagismo, hipertensão, antecedente familiar de DAC prematura e micro ou macroalbuminúria), além do próprio diabetes, sejam sistematicamente rastreados. Entretanto, estudo recente de rastreamento, o DIAD Study (3), mostrou que $22 \%$ dos pacientes assintomáticos têm isquemia miocárdica detectada pela cintilografia miocárdica com adenosina. Se as recomendações da ADA fossem aplicadas na população deste estudo, apenas 40\% dos pacientes com isquemia seriam diagnosticados.

Recentemente, a elevada prevalência de eventos cardiovasculares em diabéticos foi corroborada pela estimativa do risco de doença cardiovascular no tempo remanescente de vida aos 50 anos de idade (4). Este risco foi de $67,1 \%$ e $57,3 \%$ para homens e mulheres diabéticos, respectivamente. Por outro lado, esse risco para os não-diabéticos foi de apenas 30,2\% e 16,3\%, respectivamente.
Embora apenas 6\% a $8 \%$ da população adulta dos Estados Unidos seja diabética, 20\% a 30\% das síndromes coronárias agudas, com ou sem supradesnivelamento segmento ST, ocorrem em diabéticos, que, por sua vez, respondem por cerca de $50 \%$ de todos os óbitos cardiovasculares.

Além disso, a despeito de terem infarto do miocárdio de extensão similar, os pacientes diabéticos têm mortalidade duas a três vezes maior do que os não-diabéticos $(5,6)$.

Portanto, a despeito de não haver recomendação de diretrizes baseadas em evidências, o rastreamento de DAC em pacientes assintomáticos é comumente praticado pelos médicos. Neste sentido, a indicação de angiografia coronária nos diabéticos costuma ser mais ampla do que nos não-diabéticos, tanto nos pacientes assintomáticos como nos sintomáticos, seja na forma de angina estável ou na forma de síndrome coronária aguda (7).

A angiografia coronária tem o objetivo de definir a presença de lesões obstrutivas graves (estenoses > 50\% a 70\%), a extensão do envolvimento arterial da doença (número de vasos com estenose) e o estado da função sistólica do ventrículo. O conhecimento destas três informações permite estimar o prognóstico e definir se, além do tratamento clínico da doença, há necessidade de procedimento de revascularização miocárdica (angioplastia ou cirurgia).

\section{CARACTERÍSTICAS ANATÔMICAS E ANGIOGRÁ- FICAS DA DOENÇA ARTERIAL CORONÁRIA NO DIABETES MELLITUS}

A extensão anatômica e as características morfológicas da aterosclerose coronária nos diabéticos têm sido comparadas com as dos não-diabéticos em vários estudos, cujos resultados são variados (8-15). Entretanto, a maioria dos relatos angiográficos mostra que as artérias coronárias ateroscleróticas dos diabéticos tem menor diâmetro e doença com distribuição mais difusa. A extensão da DAC é habitualmente expressa em termos de número de artérias com estenose significante (> 50\% a 70\%) pela angiografia. Isso permite classificar a DAC em uni-, bi- ou tri-arterial. Entretanto, a extensão pode ser expressa também em termos de carga de placa aterosclerótica, definida por ultra-som intracoronário (USIC), como a soma da área da placa com a área da camada média dividida pela área de secção transversal do vaso, num determinado ponto. Este critério não leva em consideração o grau de estenose e mostra que a carga de placa dos diabéticos 
é maior do que a dos não-diabéticos. Este aspecto morfológico não é devidamente verificado pela angiografia, porque esta nada mais é do que uma "luminografia" da artéria, que por sua vez se modifica apenas quando há estenose. Assim sendo, o estudo angiográfico do Coronary Artery Surgery Study (CASS), com mais de 15.000 pacientes, mostrou apenas uma modesta correlação entre extensão da DAC com a presença do diabetes (9). Entretanto, utilizando-se do recurso da análise angiográfica quantitativa, dois outros estudos mostraram doença mais grave nesses pacientes $(13,14)$.

Recentemente, nosso grupo descreveu que mulheres diabéticas submetidas a angiografia por síndrome coronária aguda sem supradesnivelamento do segmento ST, em comparação com as não-diabéticas, têm não só mais doença tri-arterial grave (estenose $>70 \%$ ) $[27,4 \%$ vs. $10,6 \%(\mathrm{p}<0,001)]$ como também maior número de vasos com estenoses $<50 \%[36,3 \%$ vs. $8,7 \%$ $(p<0,001)](15)$. Estas observações corroboraram as de outro importante estudo, no qual pacientes diabéticos com DAC apresentaram escore de comprometimento coronário mais alto (a soma de todas as lesões ateroscleróticas detectadas) que os não-diabéticos: 352 \pm 232 vs. $211 \pm 201$ unidades $(\mathrm{p}<0,0001)$, respectivamente (12).

Um estudo de angioscopia coronária em pacientes com angina instável descreveu que placas ulceradas estão presentes em $94 \%$ dos pacientes diabéticos e em apenas $60 \%$ dos não-diabéticos $(\mathrm{p}<0,01)$. Por outro lado, trombo intracoronário foi visto em $94 \%$ dos diabéticos e em apenas $55 \%$ dos não-diabéticos $(\mathrm{p}<0,004)$ (16). Isto está de acordo com o conhecimento atual de que estenoses de grau leve a moderado $(<50 \%$ a $70 \%)$ são a principais causadoras de síndromes coronárias agudas, simplesmente porque elas são muito mais freqüentes do que as estenoses mais graves $(17,18)$. Isto permite entender por que doença mais difusa tem prognóstico pior do que doença mais localizada, mesmo com estenose mais grave.

Uma outra contribuição do nosso grupo foi a caracterização angiográfica e através do USIC, da DAC no diabético tipo 1 com insuficiência renal crônica terminal em hemodiálise (19). Esses pacientes, quando submetidos a cateterismo na avaliação pré-operatória de transplante de rim ou duplo (rim e pâncreas), têm prevalência de DAC grave (estenose > 50\%) de 58\%. Saliente-se que a idade mediana desses pacientes era de 33,5 anos, a mediana da duração do diabetes era de 21 anos e a mediana do tempo de diálise era de 23 meses. Uma investigação adicional mostrou que a aterosclerose coronária desses pacientes tem distribuição muito difusa, estando presente, pelo USIC, em todos os segmentos proximais dos três vasos principais. Ainda, encontramos um predomínio de remodelamento vascular intermediário e de placas fibróticas (20).

Muito provavelmente, esses aspectos morfológicos e quantitativos da DAC nos diabéticos estão relacionados ao seu pior prognóstico e à evolução clínica desfavorável com ambas a técnicas de revascularização miocárdica, a saber, angioplastia (21) e cirurgia (22). Essa evolução desfavorável é secundária mais à progressão da doença do que à falência primária ou técnica da revascularização.

\section{REVASCULARIZAÇÃO MIOCÁRDICA DA DOENÇA ARTERIAL ÇORONÁRIA NO DIABETES MELLITUS: INDICAÇÕES E RESULTADOS DA ANGIOPLASTIA E CIRURGIA}

A cirurgia foi a primeira modalidade de revascularização disponível para tratar a DAC. Três grandes estudos multicêntricos randomizados (Coronary Artery Surgery Study, Veteran's Administration Coronary Artery Bypass Trial e European Coronary Artery Bypass Trial) foram realizados, na década de oitenta, para comparar a cirurgia com o tratamento clínico (23). Estes estudos mostraram que a cirurgia é mais eficaz do que o tratamento clínico no controle da angina. Entretanto, o achado mais importante foi que nos pacientes com DAC mais grave (presença de estenose em mais de uma artéria, estenose do tronco da artéria coronária esquerda e com disfunção sistólica grave do ventrículo esquerdo), a cirurgia resulta em expressivo aumento da sobrevida. As observações desses três estudos estabeleceram a cirurgia de revascularização miocárdica como um dos procedimentos cirúrgicos mais realizados no mundo (24).

Em relação aos diabéticos, o estudo CASS mostrou que a cirurgia resulta em melhor sobrevida como nos não-diabéticos (25). Entretanto, estudos mais contemporâneos têm descrito que os diabéticos tratados com cirurgia têm o dobro de mortalidade $(7,8 \%$ vs. $3,6 \%)$ no seguimento de dois anos em comparação com os não-diabéticos. A mortalidade precoce (<30 dias) também foi maior (6,7\% vs. $3 \%)(26)$.

A angioplastia coronária foi introduzida em 1977, por Andreas Gruentzig, para promover a restauração do lúmen arterial coronário através da insuflação de um pequeno balão. O procedimento assim proposto foi inicialmente indicado para casos muito selecionados por critérios anatômicos (estenoses curtas, proximais, sem calcificação e em pacientes com função 
ventricular esquerda preservada). A otimização dos cateteres, dos balões e fios guias, e a experiência dos médicos com a nova técnica, permitiram a ampliação progressiva das indicações. Assim sendo, na segunda metade da década de oitenta e primeira metade da década de noventa, a angioplastia se tornou em um procedimento de revascularização amplamente utilizado e alternativo à cirurgia de revascularização em algumas situações. Os resultados clínicos eram caracterizados por mortalidade de 1-2\% e complicação do acesso vascular de 3\%. Entretanto, a segurança do procedimento era comprometida pela ocorrência de oclusão aguda da artéria tratada, que resultava em cirurgia de revascularização miocárdica de urgência em cerca de $4 \%$ dos procedimentos. Outra limitação era a recorrência de angina por retorno da estenose, que resultava em necessidade de um novo procedimento de revascularização em $20-30 \%$ dos casos.

A despeito dessas limitações, a utilização da angioplastia avançou bastante e determinou a realização de vários estudos multicêntricos randomizados para compará-la com a cirurgia. Um dos mais importantes estudos, o Bypass Angioplasty Revascularization Investigation (BARI), mostrou marcada superioridade da cirurgia, com mortalidade de 5,8\% aos 5,4 anos de seguimento, contra $20,6 \%$ de mortalidade com a angioplastia (27). Entretanto, essa superioridade foi limitada aos pacientes que receberam anastomose da artéria mamária interna esquerda para a artéria descendente anterior, $82 \%$ dos casos, e aos diabéticos, $25 \%$ dos casos (28).

A despeito da menor mortalidade tardia com a cirurgia no estudo BARI, a prática clínica não incorporou essa mensagem pelas seguintes razões: grandes registros e estudos randomizados subseqüentes demonstraram não haver diferença de mortalidade, houve aprimoramento técnico e tecnológico da angioplastia e pacientes e clínicos estão cada vez mais resistentes a procedimentos cirúrgicos de grande porte.

A principal limitação da angioplastia com o uso rotineiro de stent convencional nos diabéticos é a taxa de 20 a $25 \%$ de reestenose intra-stent, comparada com 10 a 15\% nos não-diabéticos (29-31). Esta limitação foi superada com introdução dos stents farmacológicos, que reduziram a taxa de reestenose clínica para 5 a $10 \%$, tanto nos diabéticos como nos não-diabéticos $(32,33)$.

Mesmo com os stents farmacológicos, a definição da melhor opção de revascularização miocárdica nos diabéticos, angioplastia ou cirurgia, constitui uma das mais importantes controvérsias da cardiologia contemporânea (34).
Assim sendo, a melhor modalidade de revascularização da DAC no diabetes tipo 2 está sendo extensivamente investigada no mais importante estudo multicêntrico, randomizado, já realizado, com a participação de vários centros brasileiros, e financiado pelo governo americano. Este ensaio clínico, denominado FREEDOM (Future REvascularization Evaluation in patients with Diabetes mellitus: Optimal management of Multivessel disease), já iniciado, vai comparar a cirurgia de revascularização miocárdica (com ou sem circulação extra-corpórea) com a angioplastia coronariana com implante de stent farmacológico (CYPHER ou TAXUS) e terapia anti-plaquetária adjunta otimizada (abciximab). Estão sendo randomizados 2.400 diabéticos com DAC multiarterial estável e elegíveis para ambas as modalidades de revascularização. É notável, neste ensaio clínico, que os pacientes de ambos os grupos receberão tratamento clínico otimizado $\left(\mathrm{HbA}_{\mathrm{lc}}\right.$ alvo $<7 \%$, LDLcolesterol $<70 \mathrm{mg} / \mathrm{dL}$, pressão arterial $<130 / 80$ $\mathrm{mmHg}$, clopidogrel por um ano e aspirina indefinidamente). O desfecho primário (composto de morte, infarto e acidente vascular cerebral) será analisado após seguimento mínimo de três anos. Enquanto o resultado de estudos como este não está disponível, tem havido recomendações nacionais e internacionais para que pacientes diabéticos submetidos a angioplastia coronariana sejam tratados com implante de stents farmacológicos liberadores de sirolimus (CYPHER) ou paclitaxel (TAXUS). Outro estudo multicêntrico está contemplando os pacientes diabéticos assintomáticos ou estáveis com sintomas leves e que estão sendo randomizados para revascularização (angioplastia ou cirurgia conforme decisão médica) ou tratamento clínico (BARI 2D) (35).

A despeito das controvérsias, a prática médica atual é balanceada por um conjunto de variáveis na decisão sobre a melhor alternativa de revascularização. Condições que favorecem a angioplastia:

- Estenoses curtas $(<20 \mathrm{~mm})$

-Vasos com diâmetro $\geq 2,8 \mathrm{~mm}$

- Ausência de estenose grave na artéria descendente anterior

- Cirurgia de revascularização miocárdica prévia

- Risco cirúrgico elevado por co-morbidades (doença renal crônica, doença cerebrovascular, doença pulmonar obstrutiva crônica)

Condições que favorecem a indicação de cirurgia:

- Estenoses longas (> $20 \mathrm{~mm}$ )

- Vasos com diâmetro $<2,8 \mathrm{~mm}$

- Presença de estenose grave da artéria descendente anterior

- Cirurgia valvar associada 
Diferentes registros da prática médica mostram que o respeito a essas considerações faz com que 20 a $25 \%$ dos pacientes submetidos a angioplastia e 30 a $35 \%$ dos pacientes submetidos a cirurgia sejam diabéticos.

Finalmente, deve-se ter em mente que qualquer procedimento de revascularização só terá eficácia plena se for associado ao tratamento clínico correto, com adesão integral às diretrizes pertinentes ao controle do diabetes, da hipertensão, da dislipidemia.

\section{REFERÊNCIAS}

1. American Diabetes Association. Consensus development conference on the diagnosis of coronary heart disease in people with diabetes: 10-11 February 1998, Miami, Florida. Diabetes Care 1998;21:1551-9.

2. Standards of medical care in diabetes - 2006. Position statement. American Diabetes Association. Diabetes Care 2006;29(suppl 1):S4-S42.

3. Wackers FJT, Young LH, Inzucchi SE, Chyun DA, Davey JA, Barrett EJ, et al.; for Detection of Ischemia in Asymptomatic Diabetics Investigators. Detection of silent myocardial ischemia in asymptomatic diabetic subjects: the DIAD study. Diabetes Care 2004;27:1954-61.

4. Lloyd-Jones DM, Leip EP, Larson MG, D'Agostino RB, Beiser A, Wilson PW, et al. Prediction of lifetime risk for cardiovascular disease by risk factor burden at 50 years of age. Circulation 2006;113:791-8.

5. Malmberg K, Rydén L. Myocardial infarction in patients with diabetes mellitus. Eur Heart J 1988;9:259-64.

6. Ryter L, Troelsen S, Beck-Nielsen H. Prevalence and mortality of acute myocardial infarction in patients with diabetes. Diabetes Care 1985;8:230-4.

7. Scanlon PJ, Faxon DP, Audet AM, Carabello B, Dehmer GJ, Eagle KA, et al. ACC/AHA guidelines for coronary angiography: executive summary and recommendations. a report of the American College of Cardiology/American Heart Association task force on practice guidelines. Circulation 1999;99:2345-57.

8. Abadie E, Masquet C, Guiomard A, Passa P. Coronary angiography in diabetic and non-diabetic patients with severe ischaemic heart disease. Diabetes Metab 1983;9:53-7.

9. Alderman EL, Corley SD, Fisher LD, Chaitman BR, Faxon DP, Foster ED, et al. Five-year angiographic follow-up of factors associated with progression of coronary artery disease in the coronary artery surgery study (CASS). CASS participating investigators and staff. J Am Coll Cardiol 1993;22:1141-54.

10. Dortimer AC, Shenoy PN, Shiroff RA, Leaman DM, Babb JD, Liedtke AJ, et al. Diffuse coronary artery disease in diabetic patients: fact or fiction? Circulation 1978;57:133-6.

11. Melidonis A, Dimopoulos V, Lempidakis E, Hatzissavas J, Kouvaras G, Stefanidis A, et al. Angiographic study of coronary artery disease in diabetic patients in comparison with non diabetic patients. Angiology 1999;50:997-1006.

12. Natali A, Vichi S, Landi P, Severi S, L'Abbate A, Ferrannini E. Coronary atherosclerosis in type II diabetes: angiographic findings and clinical outcome. Diabetologia 2000;43:63241.

13. Pajunen P, Nieminen MS, Taskinen MR, Syvanne M. Quantitative comparison of angiographic characteristics of coronary artery disease in patients with non insulin-dependent diabetes mellitus compared with matched non diabetic control subjects. Am J Cardiol 1997;80:550-6.

14. Thomas CS, Cherian G, Hayat NJ, Varma LK. Angiographic comparison of coronary artery disease in Arab women with and without type II diabetes mellitus. Med Princ Pract 2002;11(suppl 2):63-8.
15. Sousa JMA, Herrman JLV, Teodoro M, Diogo S, Terceiro BB, Paola AA, et al. Comparação da coronariografia de mulheres diabéticas e não-diabéticas com síndrome coronariana aguda sem supradesnivelamento de ST. Arq Bras Cardiol 2006;86:150-5.

16. Silva JA, Escobar A, Collins TJ, Ramee SR, White CJ. Unstable angina: a comparison of angioscopic findings between diabetic and non diabetic patients. Circulation 1995;92:1731-6.

17. Davies MJ. Stability and instability: two faces of coronary atherosclerosis. Paul Dudley white lecture 1995. Circulation 1996;94:2013-20.

18. Davies MJ, Bland JM, Hangartner JRW, Angelini A, Thomas AC. Factors influencing the presence or absence of acute coronary artery thrombi in sudden ischaemic death. Eur Heart J 1989;10: 203-8.

19. Oliveira DC, Gusmão Filho G, Nakamoto A, Souza FL, Sa JR, Pestana JO, et al. Prevalência da doença arterial coronária em diabéticos tipo 1 candidatos a transplante duplo (rim e pâncreas). Arq Bras Cardiol 2005;84:108-10.

20. Oliveira DC. Prevalência e características angiográficas e ultra-sonográficas da doença arterial coronariana em diabéticos tipo $1 \mathrm{com}$ insuficiência renal crônica em hemodiálise. Tese apresentada à Escola Paulista de Medicina, Universidade Federal de São Paulo para obtenção do título de Doutor em Ciências, 2005.

21. Cantor WJ, Miller JM, Hellkamp AS, Kramer JM, Peterson ED, Hasselblad V, et al. Role of target vessel size and body surface area on outcomes after percutaneous coronary interventions in women. Am Heart J 2002;144:297-302.

22. O'Connor NJ, Morton JR, Birkmeyer JD, Olmstead EM, $\mathrm{O}^{\prime}$ Connor GT. Effect of coronary artery diameter in patients undergoing coronary bypass surgery. Northern New England cardiovascular disease study group. Circulation 1996;93:652-5.

23. Flaherty JD, Davidson CJ. Diabetes and coronary revascularization. JAMA 2005;293:1501-8.

24. Eagle KA, Guyton RA, Davidoff R, Ewy GA, Fonger J, Gardner TJ, et al. ACC/AHA 2004 guideline update for coronary artery bypass graft surgery: a report of the American College of Cardiology/American Heart Association Task Force on Practice Guidelines (Committee to Update the 1999 Guidelines for Coronary Artery Bypass Graft Surgery). Circulation 1999;100:1464-80.

25. Barzilay JI, Kronmal RA, Bittner V, Eaker E, Evans C, Foster ED. Coronary artery disease and coronary artery bypass grafting in diabetic patients aged 65 years or more: a report from the Coronary Artery Surgery Study (CASS) Registry. Am J Cardiol 1994;74:334-9.

26. Herlitz J, Wognsen GB, Emanuelsson H, Haglid M, Karlson BW, Karlsson T, et al. Mortality and morbidity in diabetic and nondiabetic patients during a 2-year period after coronary artery bypass grafting. Diabetes Care 1996;19:698-703.

27. The Bypass Angioplasty Revascularization Investigation (BARI) Investigators. Comparison of coronary bypass and angioplasty in patients with multivessel disease. N Engl J Med 1996;335:217-25.

28. Brooks MM, Jones RH, Bach RG, Chaitman BR, Kern MJ, Orszulak TA, et al. Predictors of mortality and mortality from cardiac causes in the Bypass Angioplasty Revascularization Investigation (BARI) randomized trial and registry. For the BARI Investigators. Circulation 2000;101:2682-9.

29. Van Belle E, Bauters C, Hubert E, Bodart JC, Abolmaali K, Meurice T, et al. Restenosis rates in diabetic patients: a comparison of coronary stenting and balloon angioplasty in native coronary vessels. Circulation 1997;96:1454-60.

30. Srinivas VS, Brooks MM, Detre KM, King SB 3rd, Jacobs AK, Johnston J, et al. Contemporary percutaneous coronary intervention versus balloon angioplasty for multivessel coronary artery disease. A Comparison of the National Heart, Lung and Blood Institute Dynamic Registry and the Bypass Angioplasty Revascularization Investigation (BARI) Study. Circulation 2002; 106:1627-33. 
31. Loutfi M, Mulvihill NT, Boccalatte M, Farah B, Fajadet J, Marco J. Impact of restenosis and disease progression on clinical outcome after multivessel stenting in diabetic patients. Cathet Cardiovasc Interv 2003;58:451-4.

32. Lima VC, Mattos LAPM, Caramori PRA, Perin MA, Mangione JA, Machado BM, et al. Intervenção coronária percutânea na prática clinica: do cateter-balão aos stents farmacológicos. Recomendações da Sociedade Brasileira de Hemodinâmica e Cardiologia Intervencionista ao Sistema Único de Saúde. Arq Bras Cardiol 2006;87:e162-7.

33. Sabate M, Jiménez-Quevedo P, Angiolillo DJ, Gomez-Hospital JA, Alfonso F, Hernandez-Antolin R, et al. Randomized comparison of sirolimus-eluting stent versus standard stent for percutaneous coronary revascularization in diabetic patients. The Diabetes and Sirolimus-Eluting Stent (DIABETES) Trial. Circulation 2005; 112:2175-83.

34. Lima VC. Rastreamento, diagnóstico e revascularização da doença arterial coronária no diabetes tipo 2: Desafios supremos da medicina contemporânea, também no Brasil! Arq Bras Endocrinol Metab 2006;50:1-3.
35. Sobel BE, Frye R, Detre KM. Burgeoning dilemmas in the management of diabetes and cardiovascular disease. Rationale for the Bypass Angioplasty Revascularization Investigation 2 Diabetes (BARI 2D) Trial Circulation 2003;107:636-42.

Endereço para correspondência:

Valter C. Lima

R. Napoleão de Barros 715, térreo

04024-002 São Paulo, SP

Fax: (11) 5572-1134

E-mail: valterlima.dmed@epm.br / vlima@uol.com.br 